\title{
Computing welfare losses from data under imperfect competition with heterogeneous goods ${ }^{\text {th }}$
}

\author{
Luis C. Corchón, Galina Zudenkova* \\ Department of Economics, Universidad Carlos III de Madrid, c/Madrid 126, 28903 Getafe, Madrid, Spain
}

\section{A R T I C L E I N F O}

\section{Article history:}

Received 4 November 2008

Received in revised form 23 April 2009

Accepted 26 April 2009

Available online 12 May 2009

\section{JEL classification}

D61

L11

L13

L50

\section{Keywords:}

Welfare losses

Product differentiation

Cournot equilibrium

Bertrand equilibrium

Spence model

\begin{abstract}
A B S T R A C T
We study the percentage of welfare losses (PWL) yielded by imperfect competition under product differentiation. When demand is linear, even if prices, outputs, costs and the number of firms can be observed, PWL is arbitrary in both Cournot and Bertrand equilibria. If in addition the elasticity of demand (resp. cross elasticity of demand) is known, we can calculate PWL in a Cournot (resp. Bertrand) equilibrium. When demand is isoelastic and there are many firms, PWL can be computed from prices, outputs, costs and the number of firms. We find that price marginal cost margins and demand elasticities may influence PWL in a counterintuitive way. We also provide conditions under which PWL increases or decreases with concentration.
\end{abstract}

\section{Introduction}

One of the most robust findings of Industrial Organization theory is that market equilibrium very often yields inefficient allocations. But how large are these inefficiencies? This topic has inspired a con siderable amount of empirical research, from the paper by Harberger (1954) to the work of Cowling and Mueller (1978), among many others.

In contrast, the theoretical literature is sparse and focuses on the case of homogeneous products. In that case, when demand and costs are linear and firms are identical, it is well known that the percentage of welfare losses (PWL) in a Cournot Equilibrium is $\frac{1}{(1+n)^{2}}$ where $n$ is the number of firms. McHardy (2000) showed that when demand is quadratic, welfare losses can be $30 \%$ larger than in the linear model. Anderson and Renault (2003) calculated PWL for a more general class

\footnotetext{
We thank Paco Alcala, Carmen Beviá, Luis Cabral, Marc Escrihuela, Joshua Gans, Larry Kranich, Rafael Moner-Colonques, Manuel Santos, Guofu Tan, Rui Zhao, two anonymous referees and the participants of seminars and conferences at the School of Business, University of Miami, SUNY at Albany, NASS, Stern School of Business, NYU, USC, UCSB and JEI 2008 at Reus for very helpful comments. The first author acknowledges the hospitality of the Department of Economics, Stern School of Business, NYU and financial support from SEJ2005-06167/ECON. The second author acknowledges financial support from BES-2005-10017.

* Corresponding author.

E-mail address: galina.zudenkova@uc3m.es (G. Zudenkova).
}

of demand functions. Johari and Tsitsiklis (2005) showed that if average costs are not increasing and the inverse demand function is concave, PWL is less than $\frac{1}{2 n+1}$. Finally, Corchón (2008) offered formulae for PWL under free entry and heterogeneous firms. He showed that PWL can be very large under these conditions. The only paper dealing with heterogeneous products is by Cable et al. (1994), who studied a linear duopoly model.

In this paper we analyze PWL in two models of imperfect competition with heterogeneous products and a representative consumer with quasi linear preferences: a model with linear demand functions, as per Dixit (1979) and Singh and Vives (1984), and a model with isoelastic demand functions, as per Spence (1976). In both models, firms produce under constant average costs.

Our first step is to find PWL as a function of the fundamentals, i.e., the parameters of the demand and cost functions. As these parameters cannot be observed, our second step is to obtain PWL as a function of observable variables: price, output, number of firms, etc. Where this is not possible, we introduce items that might be estimated such as elasticity of demand. The goal of our analysis is to study the impact of observable variables on PWL. ${ }^{1}$ Even though PWL can be calculated

${ }^{1}$ This paper does not focus on the maximal PWL. Since product differentiation reduces competition, PWL in our framework is at least as high as it would be under product homogeneity. See Footnote 7 for the case of identical firms and formulae (2.15), and (2.17) for the case of non-identical firms. 
directly from the data on a case by case basis, our approach pinpoints the theoretical factors explaining PWL.

We first consider the model with linear demand. Assume that firms and demand functions are identical. We show that, given an observation of price, output, marginal cost and the number of firms, there exist parameters of the demand function that convert this observation into a Cournot or a Bertrand equilibrium such that PWL is arbitrary (Propositions 1 and 2). This result shows that PWL is unrelated to the differences among profit rates, contrary to Harber ger's dictum: "The differences among these profit rates, as between industries, give a broad indication of the extent of resource malallocation" (op. cit. p. 79). In our model all firms have the same rate of return on capital but PWL can be high. It seems that Harberger's procedure picks up welfare losses stemming from the failure of markets to equalize profit rates, and not welfare losses from oligopolistic misallocation. The issues are related, but distinct.

Next we show that if the elasticity of demand can be estimated, PWL in a Cournot equilibrium can be computed from observables (Proposition 3). The elasticity of demand does not add any new information in the case of a Bertrand equilibrium because it can be obtained from the markup and the first order condition of profit maximization. We show that if the cross elasticity of demand can be estimated, PWL can be computed from observations (Proposition 5). Finally, we study how PWL depends on these variables (Propositions 4 and 6). Some results are as expected, but others are not: PWL is decreasing on the price marginal cost margins (often referred to as the "monopoly index", Lerner, 1934), for example, in both Cournot and Bertrand equilibria. ${ }^{2}$ Another surprising result is that PWL increases with the elasticity of demand in a Bertrand equilibrium. Why is this so? Consider two markets, $\mathrm{A}$ and $\mathrm{B}$, and let the price marginal cost margin be larger in $A$ than in $B$. This means that the triangle that represents welfare losses is larger in $A$ than in $B$. However, the realized welfare is also larger in $A$ than in $B$ because the demand function in $A$ is above the demand function in B. A priori, there is no good reason to expect that one effect is larger than the other. In fact, as we noted before, when costs and demand are linear and firms are identical, these two effects cancel each other out and PWL only depends on the number of firms. ${ }^{3}$ The same argument goes for demand elasticity: a larger demand elasticity means less welfare losses and less realized welfare, so the total effect is ambiguous.

Next we introduce heterogeneity in demand and costs. We focus on the relationship between concentration and welfare losses. Some papers have found that the Hirschman Herfindahl $(H)$ index of concentration is not a good measure of welfare losses. Daughety (1990) came to this conclusion because more concentration may be associated with a larger output in a leader follower equilibrium. In papers by Farrell and Shapiro (1990), Cable et al. (1994) and Corchón (2008), the same result was related to the fact that the firms could be of different sizes. ${ }^{4}$ This finding contrasts with the 1992 Merger Guidelines issued by the Federal Trade Commission (FTC), where $H$ is considered a reasonable measure of welfare losses (Coate, 2005). We show that when it is optimal to allow all firms to produce, PWL increases with $H$ in both Cournot and Bertrand equilibria (Proposi tion 7). This case arises when goods are poor substitutes. We also show that when it is optimal to allow only one firm to produce, PWL decreases with $H$. This is what happened in the papers cited above where products are perfect substitutes.

\footnotetext{
2 This was noted by Formby and Layson (1982) in the case of monopoly.

3 In other words, price-marginal cost margins do not control for the size of demand. Thus, a high margin might indicate either that demand is very large and firms are having good times-even if they are very competitive-or that firms are "exploiting" consumers and destroying a large part of the surplus. This is true even if actual production is known, because the price-marginal cost margin is a poor indicator of efficient production.

${ }^{4}$ The point that minor firms may be harmful for welfare was first made by Lahiri and Ono (1988).
}

Thus, we find that concentration is bad (good) for welfare when goods are poor (good) substitutes. The reason is that efficient production must balance cost savings against consumer satisfaction. The former favors concentrating production in the most efficient firms, while the latter may require considerable diversification of production. If the last effect is not very large (i.e., when the products are close substitutes), cost savings drive efficiency and thus concentration does not harm efficiency. If the products are poor substitutes, however, efficient production requires output dispersion so concentration is harmful. We also show that at the value of $H$ proposed by the FTC as a threshold for a concentrated industry, PWL is large in a Cournot equilibrium but may be small in a Bertrand equilibrium. ${ }^{5}$

In Section 3 we assume that the representative consumer has preferences representable by a CES utility function. We also assume a large number of identical firms. This model (Spence, 1976) and its variants (e.g. Dixit and Stiglitz, 1977) are popular in the fields of monopolistic competition, international trade, geography and eco nomics. We depart from these models, however, by assuming that the number of firms is exogenous. The reason for this difference is that to endogenize the number of firms we need fixed costs, which may produce large PWL (Corchón, 2008). Since in this paper we want to focus on the PWL produced by product heterogeneity, we must assume that the number of firms is given. We show that PWL tends to zero as demand elasticity tends to infinity, and that PWL tends to one as the degree of homogeneity of the CES function tends to one (Proposition 8). This result qualifies a conjecture of Stigler (1949): “... the predictions of this standard model of imperfect competition differ only in unimportant respects from those of the theory of competition because the underlying conditions will usually be accompanied by very high demand elasticities for the individual firms". Although a high elasticity of demand makes PWL small in this model, given any elasticity of demand we can obtain a PWL as close to one as we wish.

Next, we show that PWL can be recovered from an observation of the price, output, marginal cost and number of firms (Proposition 9). However, a low price marginal cost margin does not guarantee that PWL is small; even if the price tends to the marginal cost, when the number of firms is sufficiently large, PWL may exceed that obtained in a linear model under monopoly. Moreover, when the number of firms tends to infinity, PWL is decreasing in the price marginal cost margin (Proposition 10). This is another case where price marginal cost margins and welfare losses are not related in the way we had previously thought.

Summing up, we have three main conclusions. First, our main message is positive: obtaining PWL from data is possible in two well known models of imperfect competition. Second, the roles of rates of returns, markups and the elasticity of demand on PWL are not always what they have been thought to be. Finally, we explain the role of the $H$ index. Our formulae unify previous views on the role of elasticities, markups and concentration in a precise way, with results that may be useful for policy making.

\section{The linear model}

In this section we assume that inverse demand is linear and that goods are substitutes. ${ }^{6}$ In the first subsection we assume that all firms are identical, which allows for clean formulae of welfare losses. In the second subsection we study the case where costs and the intercepts of inverse demands vary among firms. The resulting formulae for PWL will then be used to discuss the role of concentration in oligopolistic markets.

\footnotetext{
${ }^{5}$ Despite the fact that, as shown by Amir and Jin (2001), $H$ is always higher in a Bertrand equilibrium than in a Cournot equilibrium.

${ }^{6}$ We study the case of complements in a companion working paper (Corchón and Zudenkova, 2008).
} 


\subsection{The symmetric case}

The market is composed of $n$ firms. The output and price of firm $i$ are denoted by $x_{i}$ and $p_{i}$ respectively. The firms are identical, sharing the cost function $c x_{i}$. There is a representative consumer with a quadratic utility function

$U=\alpha \sum_{i}^{n} x_{i} \quad \frac{\beta}{2} \sum_{i}^{n} x_{i}^{2} \quad \frac{\gamma}{2} \sum_{i}^{n} x_{i} \sum_{j \neq i} x_{j}+M, \alpha>c, \beta>\gamma \geq 0$,

where $M$ is the consumption of an outside good which is the numeraire. The budget constraint is $\sum_{i}{ }_{1} p_{i} x_{i}+M=I$, where $I$ is a given income. Substituting $M$ into $U$ and eliminating $I$ (which is constant), we obtain $U=\alpha \sum_{i}^{n}{ }_{1} x_{i} \quad \frac{\beta}{2} \sum_{i}^{n}{ }_{1} x_{i}^{2} \quad \frac{\gamma}{2} \sum_{i}^{n}{ }_{1} x_{i} \sum_{j \neq i} x_{j} \quad \sum_{i}^{n}{ }_{1} p_{i} x_{i}$. We call this quantity the consumer surplus. Under our assumptions, this function is concave. The first order condition (FOC) of consumer surplus maximization yields $p_{i}=\alpha \quad \beta x_{i} \quad \gamma \sum_{j \neq i} x_{j}, i=1,2 \ldots, n$. If $\gamma=0$ the products are independent, while if $\gamma \simeq \beta$ they are almost perfect substitutes.

Definition 1. A linear market is a list $\{\alpha, \beta, \gamma, c, n\}$ with $\alpha>c, \beta>\gamma \geq 0$ and $n \in \mathbb{N}$.

Social welfare is defined as

$W=\alpha \sum_{i}^{n} x_{i} \quad \frac{\beta}{2} \sum_{i}^{n} x_{i}^{2} \quad \frac{\gamma}{2} \sum_{i}^{n} x_{i} \sum_{j \neq i} x_{j} \quad c \sum_{i}^{n} x_{i}$.

The social optimum is a list of outputs that maximize social welfare. It is easy to see that the optimal outputs $x_{i}^{o}$ (which are all identical) and the social welfare in the optimum $W^{o}$ are

$x_{i}^{o}=\frac{\alpha \quad c}{\beta+\gamma\left(\begin{array}{ll}n & 1\end{array}\right)}$ and $W^{o}=\frac{n\left(\begin{array}{ll}\alpha & c\end{array}\right)^{2}}{2\left(\beta+\left(\begin{array}{ll}n & 1\end{array}\right) \gamma\right)}$.

Now we are ready to define our equilibrium concepts.

Definition 2. A Cournot equilibrium in a linear market is a list of outputs $\left(x_{1}^{c}, x_{2}^{c}, \ldots, x_{n}^{c}\right)$ such that for each $i, x_{i}^{c}$ maximizes $\left(\begin{array}{llll}\alpha & \beta x_{i} & \gamma \sum_{j \neq i} x_{j}^{c} & c\end{array}\right) x_{i}$.

From the FOC of profit maximization we find that

$x_{i}^{c}=\frac{\alpha \quad c}{2 \beta+\gamma(n \quad 1)}, i=1,2, \ldots, n$

In order to define a Bertrand equilibrium we write the demand for firm $i$ :

$x_{i}=\frac{\alpha(\beta \quad \gamma) \quad p_{i}(\beta+\gamma(n \quad 2))+\gamma \sum_{j \neq i} p_{j}}{(\beta \quad \gamma)(\beta+\gamma(n \quad 1))}$

$\equiv x_{i}^{b}\left(p_{i}, p \quad{ }_{i}\right), i=1,2, \ldots, n$

where $p_{-i}$ is a list of all prices minus $p_{i}$. Now we can define a Bertrand equilibrium.

Definition 3. A Bertrand equilibrium in a linear market is a list of prices $\left(p_{1}^{b}, p_{2}^{b}, \ldots, p_{n}^{b}\right)$ such that for each $i, p_{i}^{b}$ maximizes $\left(p_{i} c\right) x_{i}^{b}\left(p_{i}, p_{-i}^{b}\right)$.

From the FOC of profit maximization we obtain

$p_{i}^{b}=\frac{\alpha(\beta \quad \gamma)+c(\beta+\gamma(n \quad 2))}{2 \beta+\gamma(n \quad 3)}, i=1,2, \ldots, n$.

Let $W^{c}$ be social welfare evaluated at the Cournot equilibrium. Let us define the percentage of welfare losses in a Cournot equilibrium as

$P W L^{c} \equiv \frac{W^{0} \quad W^{c}}{W^{0}}$.
Lemma 1. In linear markets the percentage of welfare losses in Cournot equilibrium is

$$
P W L=\frac{1}{\left(2+\left(\begin{array}{ll}
n & 1
\end{array}\right) \frac{\gamma}{\beta}\right)^{2}}
$$

Proof. From (2.1), social welfare in a Cournot equilibrium can be written as $W^{c}=\alpha n x_{i}^{c} \quad \frac{\beta}{2} n x_{i}^{c 2} \quad \frac{\gamma}{2} n\left(\begin{array}{lll}n & 1\end{array}\right) x_{i}^{c 2} \quad c n x_{i}^{c}$. Thus, from (2.2) we obtain

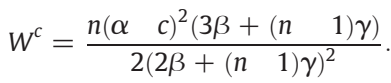

Then,

$P W L^{c}=1 \quad \frac{W^{c}}{W^{0}}=\frac{1}{\left(\begin{array}{ll}2+\left(\begin{array}{ll}n & 1\end{array}\right) \frac{\gamma}{\beta}\end{array}\right)^{2}}$.

Notice that $P W L^{c}$ is decreasing in the degree of product dif ferentiation, $\frac{\gamma}{\beta}$. Thus, the minimal $P W L^{c}$ is $\frac{1}{(n+1)^{2}}$ and occurs when $\gamma \simeq \beta$, i.e., when the products are perfect substitutes. The maximal $P W L^{c}$ is equal to 0.25 and occurs for the minimal value of $\frac{\gamma}{\beta}$, which is zero when products are independent.

The following Lemma derives $P W L^{b}$, the percentage of welfare losses in a Bertrand equilibrium.

Lemma 2. In linear markets the percentage of welfare losses in Bertrand equilibrium is

$P W L^{b}=\left(\frac{1 \quad \frac{\gamma}{\beta}}{2+(n-3) \frac{\gamma}{\beta}}\right)^{2}$.

Proof. From (2.4) we find that all firms produce the same output $x_{i}^{b}$, namely

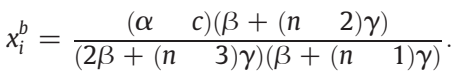

Social welfare in a Bertrand equilibrium is $W^{b}=\alpha n x_{i}^{b} \quad \frac{\beta}{2} n x_{i}^{b 2}$ $\frac{\gamma}{2} n(n \quad 1) x_{i}^{b 2} \quad$ cn $x_{i}^{b}$, or

$W^{b}=\frac{n\left(\begin{array}{ll}\alpha & c\end{array}\right)^{2}\left(3 \beta+\left(\begin{array}{ll}n & 4\end{array}\right) \gamma\right)\left(\beta+\left(\begin{array}{ll}n & 2\end{array}\right) \gamma\right)}{2\left(2 \beta+\left(\begin{array}{ll}n & 3\end{array}\right) \gamma\right)^{2}\left(\beta+\left(\begin{array}{ll}n & 1\end{array}\right) \gamma\right)}$.

Thus,

$P W L^{b}=1 \quad \frac{W^{b}}{W^{0}}=\left(\begin{array}{cc}1 & \frac{\gamma}{\beta} \\ \hline 2+\left(\begin{array}{ll}n & 3\end{array}\right) & \frac{\gamma}{\beta}\end{array}\right)^{2}$.

Note that $P W L^{b}$ is decreasing in the degree of product differentia tion $\frac{\gamma}{\beta}$ Thus, minimal $P W L^{b}$ is (almost) zero and occurs when $\gamma \simeq \beta$, i.e., when the products are perfect substitutes. Maximal $P W L^{b}$ is equal to 0.25 and occurs for $\frac{\gamma}{\beta}=0$, when products are independent. Clearly, if $n=1, P W L^{j}=0.25, j=c, b$, so for the remainder of this section we will assume that $n>1$.

We are interested in the PWL yielded by imperfectly competitive markets, conditional on certain observable variables: market prices, outputs, marginal cost, and the number of firms. We assume that the marginal cost is observable because under constant returns, the marginal cost equals the average variable cost, which in principle can be observed (wages, raw materials, etc.). Formally:

Definition 4. An observation is a list $\left\{\mathfrak{p}, \mathfrak{x}_{i}, \mathfrak{c}, \mathfrak{n}\right\}$ where $\mathfrak{p}$ is the market price, $\mathfrak{x}_{i}$ is the output of firm $i, \mathfrak{c}(<\mathfrak{p})$ is the marginal cost and $\mathfrak{n}$ is the number of firms.

Let us relate PWL to the observable variables. First consider the Cournot equilibrium. 
Proposition 1. Given an observation $\left\{\mathfrak{p}, \mathfrak{x}_{i}, \mathfrak{c}, \mathfrak{n}\right\}$ and a number $\mathfrak{v} \in\left(\frac{1}{(\mathfrak{n}+1)^{2}}, 0.25\right]$ there is a linear market $\{\alpha, \beta, \gamma, \mathfrak{c}, \mathfrak{n}\}$ such that $\left(\mathfrak{x}_{i}, \mathfrak{x}_{i}, \ldots, \mathfrak{x}_{i}\right)$ is a Cournot equilibrium for this market, where $\mathfrak{p}=\alpha \quad \beta \quad \beta \mathfrak{x}_{i} \quad \gamma(\mathfrak{n} \quad 1) \mathfrak{x}_{i}$ and $P W L^{c}=\mathfrak{v}$.

Proof. Let

$\alpha=\mathfrak{c}+\frac{\mathfrak{p} \quad \mathfrak{c}}{\sqrt{ } \mathfrak{v}}, \beta=\frac{\mathfrak{p} \quad \mathfrak{c}}{\mathfrak{x}_{i}}$ and $\gamma=\frac{\left(\begin{array}{ll}\mathfrak{p} & \mathfrak{c}\end{array}\right)\left(\begin{array}{ll}1 & 2 \sqrt{ } \mathfrak{v}\end{array}\right)}{\left(\begin{array}{ll}\mathfrak{n} & 1\end{array}\right) \mathfrak{x}_{i} \sqrt{ } \mathfrak{v}}$

Clearly, $\alpha>\mathfrak{c}$ and $\beta>\gamma \geq 0$ since $\mathfrak{p}>\mathfrak{c}, \mathfrak{v}>\frac{1}{(2+1)^{2}}$ and $\mathfrak{v} \leq 0.25$. We easily see that the linear market $\{\alpha, \beta, \gamma, \mathfrak{c}, \mathfrak{n}\}$ yields an equilibrium where $x_{i}^{c}=\mathfrak{x}_{i}, i=1,2, \ldots, n, \mathfrak{p}=\alpha \quad \beta \mathfrak{x}_{i} \quad \gamma(\mathfrak{n} \quad 1) \mathfrak{x}_{i}$ and $P W L^{c}=\mathfrak{v}$, so the proof is complete.

Now we turn to the Bertrand equilibrium.

Proposition 2. Given an observation $\left\{\mathfrak{p}, \mathfrak{x}_{i}, \mathfrak{c}, \mathfrak{n}\right\}$ and a number $\mathfrak{v} \in$ $(0,0.25]$ there is a linear market $\{\alpha, \beta, \gamma, \mathfrak{c}, \mathfrak{n}\}$ such that $(\mathfrak{p}, \mathfrak{p}, \ldots, \mathfrak{p})$ is Bertrand equilibrium for this market, $\mathfrak{x}_{i}=x_{i}^{b}\left(\mathfrak{p}, \mathfrak{p} \quad{ }_{i}\right)$, where $\mathfrak{p}_{-i}$ is a list of $\mathfrak{n} \quad 1$ identical $\mathfrak{p}$ and $P W L^{b}=\mathfrak{v}$.

Proof. Let

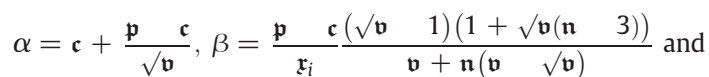
$\gamma=\frac{\mathfrak{c} \quad \mathfrak{p}}{\mathfrak{x}_{i}} \frac{1 \quad 3 \sqrt{ } \mathfrak{v}+2 \mathfrak{v}}{\mathfrak{v}+\mathfrak{n}(\mathfrak{v} \quad \sqrt{ } \mathfrak{v})}$.

It is easy to check that $0<\mathfrak{c}<\alpha$ and $\beta>\gamma \geq 0$. The linear market $\{\alpha, \beta, \gamma, \mathfrak{c}, \mathfrak{n}\}$ yields a Bertrand equilibrium with $p_{i}^{b}=\mathfrak{p}, x_{i}^{b}=\mathfrak{x}_{i}$ and $P W L^{b}=\mathfrak{v}$, so the proof is complete.

Propositions 1 and 2 show that observable variables put very few restrictions on PWL. In particular, neither price marginal cost margins nor profit rates have any relationship with PWL. Let us look for restrictions that can take a bite out of PWL. ${ }^{7}$ Suppose that the demand elasticity, denoted by $\varepsilon$, is observable. From (2.3), we have

$\varepsilon \equiv \quad \frac{\partial x_{i}}{\partial p_{i}} \frac{\mathfrak{p}}{\mathfrak{x}_{i}}=\frac{\beta+\gamma(\mathfrak{n} \quad 2)}{(\beta \quad \gamma)(\beta+\gamma(\mathfrak{n} \quad 1))} \frac{\mathfrak{p}}{\mathfrak{x}_{i}}$.

Let us introduce a new piece of notation: $\mathfrak{T} \equiv \varepsilon \frac{p \quad c}{p}$ Now we have the following result.

Proposition 3. Given an observation $\left\{\mathfrak{p}, \mathfrak{x}_{i}, \mathfrak{c}, \mathfrak{n}, \varepsilon\right\}$ such that $\mathfrak{T} \equiv$ $\varepsilon \frac{\mathfrak{p} \mathbf{c}}{\mathfrak{u}} \geq 1$, there is a linear market $\{\alpha, \beta, \gamma, \mathfrak{c}, \mathfrak{n}\}$ such that $\left(\mathfrak{x}_{i}, \mathfrak{x}_{i}, \ldots, \mathfrak{x}_{i}\right)$ is a Cournot equilibrium for this market, $\left.\mathfrak{p}=\alpha \quad \beta \quad \begin{array}{lll}\mathfrak{x}_{i} & \gamma(\mathfrak{n} & 1\end{array}\right) \mathfrak{x}_{i}$ and

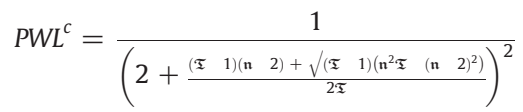

Proof. Let

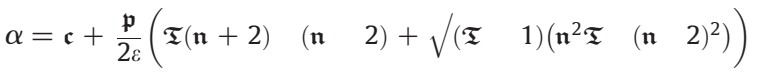

$\beta=\frac{\mathfrak{p} \quad \mathfrak{c}}{\mathfrak{x}_{i}}$

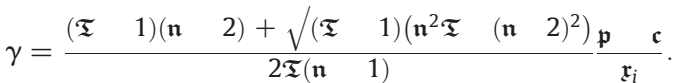

Clearly, $\beta>0$. We need to show that $0 \leq \frac{\gamma}{\beta}<1$ and $\alpha>\mathfrak{c}$. For $\mathfrak{T} \geq 1$ the square root is defined in real numbers and $\left.\sqrt{(\mathfrak{T}} \quad 1)\left(\begin{array}{lll}\mathfrak{n}^{2} \mathfrak{T} & (\mathfrak{n} & 2\end{array}\right)^{2}\right) \geq$

\footnotetext{
7 The maximum PWL in both Cournot and Bertrand equilibria occurs when $\gamma \simeq 0$,
} namely $P W L \simeq 0.25$, which corresponds to PWL under monopoly.
$\left(\begin{array}{ll}\mathfrak{T} & 1\end{array}\right)\left(\mathfrak{n} \quad\right.$ 2) because if not, $\mathfrak{n}^{2} \mathfrak{T}<(\mathfrak{n} \quad 2)^{2} \mathfrak{T}$, which is impossible. Then the condition $0 \leq \frac{\gamma}{\beta}<1$ amounts to

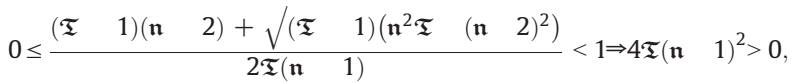

which always holds for $\mathfrak{T} \in[1, \infty)$. The condition $\alpha>\mathfrak{c}$ amounts to

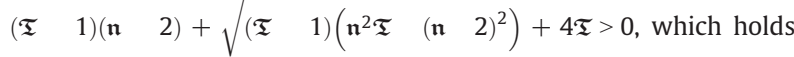
for $\mathfrak{T} \in[1, \infty)$. It is straightforward to show that the linear market $\{\alpha, \beta, \gamma, \mathfrak{c}, \mathfrak{n}\}$ yields a Cournot equilibrium where $x_{i}^{c}=\mathfrak{x}_{i}, \mathfrak{p}=\alpha \quad \beta \mathfrak{r}_{-}$ \{i\} $\gamma(\mathfrak{n} \quad 1) \mathfrak{x}_{-}\{i\}$, and $P W L^{c}$ as defined by (2.6).

According to Proposition 3 we can calculate $P W L^{c}$ from three variables: the number of firms, the elasticity of demand and the price marginal cost ratio. Let us study how $P W L^{c}$ depends on $\mathfrak{n}$ and $\mathfrak{T}$. Notice that observable variables are not independent and that in general, a variation in just one observable variable cannot be obtained by a variation of a single unobservable variable (see the first three equations of the proof of Proposition 3). Our exercise just gives us the difference in PWL between two markets in which all observables except one are identical. Thus, it emphasizes the role played by the observables, which sometimes contradicts intuition.

Proposition 4. $P W L^{c}$ is decreasing in $\mathfrak{n}$, the elasticity of demand, and the price marginal costs margins.

Proof. From (2.6) we find

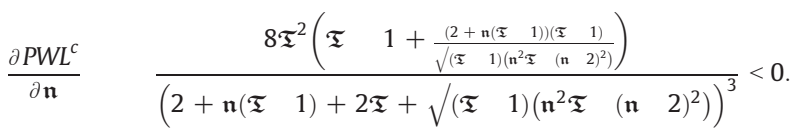

Next we compute $\frac{\partial\left(\frac{\gamma}{\beta}\right)}{\partial \mathfrak{T}}$ :

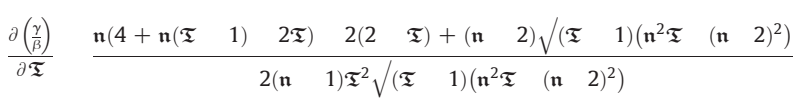

This derivative is positive, so $P W L^{c}$ decreases with $\mathfrak{T}$. $\square$

In Proposition 4, the signs of the effects of the number of firms and demand elasticity are just as expected: more competition i.e., a higher value of $\mathfrak{n}$ or $\varepsilon$ is good. However the effect of price marginal cost margins runs counter to intuition. As we remarked in the introduction, this is because the price marginal cost margin affects both welfare losses and realized welfare.

Note that we have been applying comparative statics, treating the

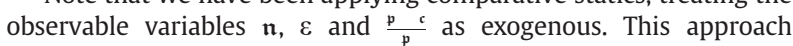
provides policy makers with a tool to predict changes in PWL due to changes in just one of the observables, taking all other variables as given. If both demand elasticity and the price marginal cost margin change, one needs to consider the comparative statics of PWL with

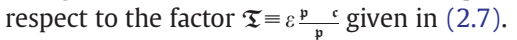

We now consider the Bertrand equilibrium. In this case, the FOC of profit maximization can be written as $p_{i}=\varepsilon\left(\begin{array}{ll}p_{i} & c\end{array}\right)$. Thus, an observation of $\varepsilon$ does not add any new information once $p_{i}$ and $c$ are observed. A way out of this problem is provided if the cross elasticity of demand $\frac{\partial x_{i}}{\partial p_{i}} \bar{p}_{i}$, denoted by $\rho$, is observable.

Proposition 5. Given an observation $\left\{\mathfrak{p}, \mathfrak{x}_{i}, \mathfrak{c}, \mathfrak{n}, \rho\right\}$ such that $\frac{\mathfrak{p}}{\mathfrak{p} \mathfrak{c}}>$ $\rho(\mathfrak{n} \quad 1) \geq 0$, there is a linear market $\{\alpha, \beta, \gamma, \mathfrak{c}, \mathfrak{n}\}$ such that $(\mathfrak{p}, \mathfrak{p}, \ldots, \mathfrak{p})$ is a Bertrand equilibrium for this market, $\mathfrak{x}_{i}=x_{i}^{b}\left(\mathfrak{p}, \mathfrak{p} \quad{ }_{i}\right)$, where $\mathfrak{p}_{-i}$ is a list of $\mathfrak{n} 1$ identical $\mathfrak{p}$ and

$\left.P W L^{b}=\left(\begin{array}{ccc}\frac{\mathfrak{p}}{\mathfrak{p} \mathfrak{c}} & \rho(\mathfrak{n} & 1\end{array}\right)\right)^{2}$. 
Proof. Let

$$
\begin{aligned}
& \alpha=\mathfrak{p}+\frac{\mathfrak{p}}{\frac{\mathfrak{p}}{\mathfrak{p} \mathfrak{c}}} \rho(\mathfrak{n} \quad 1)
\end{aligned}
$$

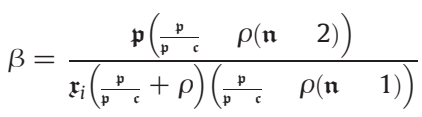

$$
\begin{aligned}
& \gamma=\frac{\mathfrak{p} \rho}{\mathfrak{x}_{i}\left(\frac{\mathfrak{p}}{\mathfrak{p} \mathfrak{c}}+\rho\right)\left(\frac{\mathfrak{p}}{\mathfrak{p} \mathfrak{c}} \quad \rho(\mathfrak{n} \quad 1)\right)}
\end{aligned}
$$

It is straightforward to prove that $\alpha>\mathbf{c}$ and $\beta>0$ and that $\beta>\gamma \geq 0$ for $\frac{\mathfrak{p}}{p}>\rho(\mathfrak{n} \quad 1) \geq 0$. One can easily show that the linear market $\{\alpha, \beta$,

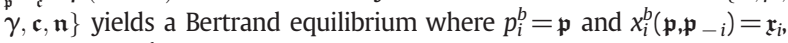
and find $P W L^{b}$ by plugging the values of $\beta$ and $\gamma$ in (2.5).

The formula (2.8) allows for the calculation of PWL in a Bertrand equilibrium from just three magnitudes: the number of firms, the price marginal cost margins (or alternatively, the elasticity of demand), and the cross elasticity of demand. Let us analyze the impact of a change in observable variables on $P W L^{b}$.

Proposition 6. $P W L^{b}$ is decreasing in the number of firms, the price marginal cost margins, and the cross elasticity of demand. $P W L^{b}$ is increasing in the elasticity of demand.

Proof. In a Bertrand equilibrium $\frac{\mathfrak{p}}{\mathfrak{p} \mathfrak{c}}=\varepsilon$. From (2.8), we therefore obtain

$\frac{\partial P W L^{b}}{\partial \mathfrak{n}}=\frac{2 \varepsilon \rho(\varepsilon \quad \rho(\mathfrak{n} \quad 1))}{\left.\left(\begin{array}{lll}2 \varepsilon & \rho(\mathfrak{n} & 1\end{array}\right)\right)^{3}}<0$,

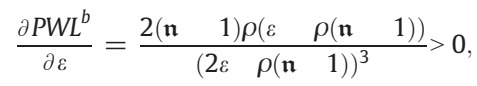

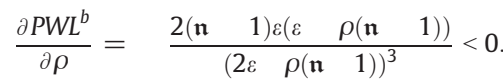

From these formulae the proposition follows. $\square$

Proposition 6 confirms our intuitions about the role of the number of firms and the cross elasticity of demand on welfare losses, namely that an increase in the number of firms decreases PWL and an increase in the cross elasticity of demand decreases PWL. The impacts of the price marginal cost margin and demand elasticity, on the other hand, are contrary to intuition. Again, we have to bear in mind that these two variables affect both welfare losses and realized welfare.

\subsection{Heterogeneous firms}

We now extend the model presented in Section 2.1 to the case where firms are heterogeneous on two counts. The marginal costs $c_{i}$ may be different for each firm $i$. The parameter $\alpha$, now denoted $\alpha_{i}$, may also vary across firms. ${ }^{8}$ Assume $\alpha_{i}>c_{i}$ for all $i$. The consumer surplus is now

$$
U=\sum_{i=1}^{n} \alpha_{i} x_{i} \quad \frac{\beta}{2} \sum_{i}^{n} x_{i}^{2} \quad \frac{\gamma}{2} \sum_{i}^{n} x_{i} \sum_{j \neq i} x_{j} \quad \sum_{i=1}^{n} p_{i} x_{i}, \beta>\gamma \geq 0
$$

\footnotetext{
${ }^{8}$ This model has been used, among others, by Häckner (2000) and Hsu and Wang (2005).
}

The restrictions below guarantee that the outputs of all firms are positive in Cournot and Bertrand equilibria.

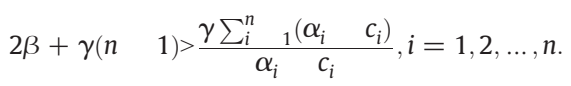

$$
\begin{aligned}
& \frac{(\beta+\gamma(n \quad 1))(2 \beta+\gamma(n \quad 3))}{\beta+\gamma(n \quad 2)}>\frac{\gamma \sum_{i}^{n}{ }_{1}\left(\alpha_{i} \quad c_{i}\right)}{\alpha_{i} \quad c_{i}} i=1,2, \ldots, n
\end{aligned}
$$

Under our assumptions, $U(\cdot)$ is concave. The FOC of consumer surplus maximization yields $p_{i}=\alpha_{i} \quad \beta x_{i} \quad \gamma \sum_{j \neq i} x_{j}, i=1,2, \ldots, n$. Social welfare is now

$W=\sum_{i}^{n} \alpha_{i} x_{i} \quad \frac{\beta}{2} \sum_{i}^{n} x_{i}^{2} \quad \frac{\gamma}{2} \sum_{i}^{n} x_{i} \sum_{j \neq i} x_{j} \quad \sum_{i}^{n} c_{i} x_{i}$.

Evaluating social welfare in the optimum is not straightforward, because it depends on the number of active firms in the optimum. For the time being, let us assume that the optimal number of active firms is $m$. Then the optimal outputs, denoted by $x_{i}^{o}$, are equal to

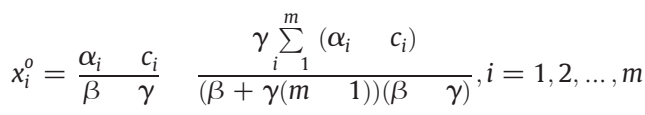

and the aggregate output in the optimum, denoted by $x^{o}$, is equal to

$x^{0}=\sum_{i}^{m} x_{i}^{o}=\frac{\sum_{i}^{m}\left(\begin{array}{ll}\alpha_{i} & c_{i}\end{array}\right)}{\beta+\gamma\left(\begin{array}{ll}m & 1\end{array}\right)}$

We now find the optimal number of firms $m$. Let us rank firms according to the value of $\alpha_{i} \quad c_{i}$. Without loss of generality assume that $\alpha_{v} \quad c_{v} \geq \alpha_{v+1} \quad c_{v+1}, v=1,2, \ldots, n \quad 1$. Clearly, if firm $v$ produces a positive output in the optimum, firms $v \quad 1, v$ 2, etc. also produce positive outputs in the optimum. Suppose that it is optimal for firms 1 through $k 1$ to produce positive outputs. By evaluating $\frac{\partial W}{\partial x_{k}}$ in (2.11) at $x_{k}=0$ and $x_{j}=x_{j}^{o}, j=1, \ldots k \quad 1$ according to (2.12), we obtain

$\frac{\partial W}{\partial x_{k}}=\alpha_{k} \quad c_{k} \quad \gamma \sum_{j}^{k} x_{j}^{o}$.

If $\frac{\partial W}{\partial x_{k}} \leq 0$, clearly, $x_{k}^{o}=0$. If $\frac{\partial W}{\partial x_{k}}>0$, firm $k$ must produce a positive output in the optimum.

This algorithm requires knowledge of all the parameters defining a market. In a companion working paper (Corchón and Zudenkova, 2008), we show that all these parameters can be recovered from market data and demand elasticities by a method identical to that applied in Propositions 3 and 5. We will focus on two particular cases. First, when $\beta\left(\begin{array}{ll}\alpha_{2} & c_{2}\end{array}\right) \leq \gamma\left(\alpha_{1} \quad c_{1}\right)$, only firm 1 will produce a positive output in the optimum since from (2.12) and (2.13), $\frac{\partial W}{\partial x_{2}}=\alpha_{2} \quad c_{2}$ $\gamma \frac{\alpha_{1} c_{1}}{\beta} \leq 0$. Second, when

$\left(\begin{array}{lll}\alpha_{n} & c_{n}\end{array}\right)(\beta+\gamma(n \quad 2))>\gamma \sum_{i}^{n} 1\left(\alpha_{i} \quad c_{i}\right)$

the number of active firms is the same in optimum and in equilibrium, because from (2.12) and (2.13), $\frac{\partial W}{\partial x_{n}}=\alpha_{n} \quad c_{n} \quad \gamma \sum_{j}^{n}{ }_{1}^{1} x_{j}^{o}>0$. Notice that the conditions in (2.9) and (2.10) are implied by (2.14).

In this framework, a Cournot equilibrium is a list of outputs $\left(x_{1}^{c}, x_{2}^{c}\right.$, $\left.\ldots, x_{n}^{c}\right)$ such that for each $i, x_{i}^{c}$ maximizes $\left(\begin{array}{lllll}\alpha_{i} & \beta x_{i} & \gamma \sum_{j \neq i} x_{j}^{c} & c_{i}\end{array}\right) x_{i}$. From the FOC of profit maximization, we obtain

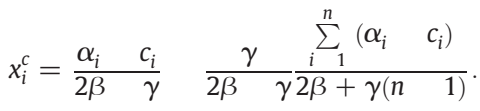


And the aggregate output at the Cournot equilibrium is

$x^{c}=\sum_{i}^{n} x_{i}^{c}=\frac{\sum_{i}^{n}\left(\alpha_{i} \quad c_{i}\right)}{2 \beta+\gamma(n \quad 1)}$.

In order to compute a Bertrand equilibrium we first write the demand for firm $i$ :

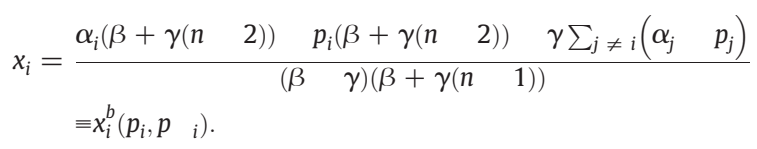

A Bertrand equilibrium is a list $\left(p_{1}^{b}, p_{2}^{b}, \ldots, p_{n}^{b}\right)$ such that for all $i p_{i}^{b}$ maximizes $\left(p_{i} \quad c_{i}\right) x_{i}^{b}\left(p_{i}, p^{b}{ }_{i}\right)$. Then we have

$$
\begin{aligned}
x_{i}^{b}= & \frac{\beta+\gamma(n \quad 2)}{(\beta \quad \gamma)(2 \beta+\gamma(2 n \quad 3))} \\
& \times\left(\begin{array}{llll}
\alpha_{i} & c_{i} & \left.\left.\gamma \frac{\beta+\gamma(n}{(2 \beta+\gamma(n} \quad 3\right)\right)(\beta+\gamma(n) & 1))
\end{array} \sum_{i}^{n}\left(\begin{array}{lll}
\alpha_{i} & c_{i}
\end{array}\right)\right)
\end{aligned}
$$

and the aggregate output at the Bertrand equilibrium is

$$
x^{b}=\frac{\beta+\gamma(n \quad 2)}{(2 \beta+\gamma(n \quad 3))(\beta+\gamma(n \quad 1))} \sum_{i}^{n}\left(\begin{array}{ll}
\alpha_{i} & \left.c_{i}\right)
\end{array}\right.
$$

Next, we link PWL to the Hirschman Herfindahl index of concentration. Let $s_{i}^{j}$ be the market share of firm $i$ in a Cournot equilibrium $(j=c)$, a Bertrand equilibrium $(j=b)$, or in the optimum $(j=0)$. We define the Hirschman Herfindahl index of concentration in either equilibrium as $H^{j} \equiv \sum_{i}{ }_{1}{ }_{1}\left(s_{i}^{j}\right)^{2}, j=c, b$. In the optimum, we define it as $H^{o} \equiv \sum_{i}{ }^{m}{ }_{1}\left(s_{i}^{o}\right)^{2}{ }^{9}$ Amir and Jin (2001) show that in our framework $H^{b}>H^{c}$.

Lemma 3. With heterogeneous firms the percentage of welfare losses in Cournot equilibrium is

$P W L^{c}=1 \quad\left(\frac{1+\frac{\gamma}{\beta}\left(\begin{array}{cc}m & 1\end{array}\right)}{m_{\bar{\beta}}^{\gamma}+\left(\begin{array}{ll}2 & \frac{\gamma}{\beta}\end{array}\right) \sum_{i}^{m}{ }_{1}^{m} s_{i}^{c}}\right)^{2} \frac{H^{c}\left(\begin{array}{ll}3 & \frac{\gamma}{\beta}\end{array}\right)+\frac{\gamma}{\beta}}{H^{o}\left(\begin{array}{ll}1 & \frac{\gamma}{\beta}\end{array}\right)+\frac{\gamma}{\beta}}$.

Proof. Social welfare in a Cournot equilibrium, denoted by $W^{c}$, is given by

$$
\begin{aligned}
W^{c} & =\sum_{i=1}^{n}\left(\alpha_{i} \quad c_{i}\right) x_{i}^{c} \quad \frac{\beta}{2} \sum_{i}^{n} x_{i}^{c 2} \quad \frac{\gamma}{2} \sum_{i}^{n} x_{i}^{c} \sum_{j \neq i} x_{j}^{c} \\
& =\frac{3 \beta}{2} H^{c} x^{c 2}+\frac{\gamma}{2} x^{c 2}
\end{aligned}
$$

Using the definition of $H^{\circ}$, social welfare in the optimum is

$W^{0}=\frac{\beta \quad \gamma}{2} H^{o} x^{02}+\frac{\gamma}{2} x^{02}$

Plugging the values of $W^{c}$ and $W^{o}$ into $P W L^{c}$ yields

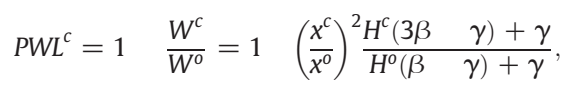

while plugging in the values of $x^{c}$ and $x^{o}$ yields formula (2.15).

\footnotetext{
${ }^{9}$ Notice that in our framework products can be imperfect substitutes, so interpreting $\sum_{i}^{n}{ }_{1}\left(s_{i}\right)^{2}$ as the Hirschman-Herfindahl index of concentration may be a bit problematic.
}

Note that $P W L^{c}$ here depends on the degree of product differentia tion $\frac{\gamma}{\beta}$, the number of active firms in the optimum $m$, the sum of the market shares of the $m$ largest firms $\sum_{i}{ }^{m}{ }_{1} s_{i}^{c}$, and the Hirschman Herfindahl indices of concentration $H^{c}$ and $H^{o}$ evaluated at the Cournot equilibrium and optimum respectively. When $m=1$ we have

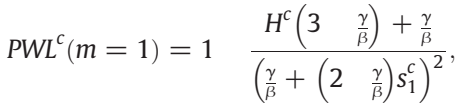

which is decreasing in $H^{c}$. In the other extreme case where $m=n$ i.e., the number of active firms is the same in the optimum and at a Cournot equilibrium we prove that

$$
P W L^{c}(m \quad n) \frac{\left.H^{c}\left(\begin{array}{ll}
1+(n & 1
\end{array}\right) \frac{\gamma}{\beta}\right) \quad \frac{\gamma}{\beta}}{H^{c}\left(\begin{array}{llll}
2 & \frac{\gamma}{\beta}
\end{array}\right)^{2}\left(\begin{array}{ll}
1+\left(\begin{array}{ll}
n & 1
\end{array}\right) \frac{\gamma}{\beta}
\end{array}\right)+\left(\frac{\gamma}{\beta}\right)^{2}\left(\begin{array}{llll}
n & 2 & \left(\begin{array}{ll}
n & 1
\end{array}\right) \frac{\gamma}{\beta}
\end{array}\right)} .
$$

If all firms are identical, $H^{c}=\frac{1}{n}$ and $P W L^{c}(m=n)=\frac{1}{\left(2+(n-1) \frac{\gamma}{\frac{\gamma}{2}}\right)^{2}}$, as in Lemma 1. Notice that $H^{c}$ and $\frac{\gamma}{\beta}$ are less than one, so for reasonable values of $n$ it makes sense to evaluate (2.16) as if $n$ were a large number. In this case (2.16) simplifies to

$$
\begin{aligned}
& P W L^{c}(m=n, n \text { large })=\frac{H^{c}}{H^{c}\left(\begin{array}{ll}
2 & \frac{\gamma}{\beta}
\end{array}\right)^{2}+\frac{\gamma}{\beta}\left(\begin{array}{ll}
1 & \frac{\gamma}{\beta}
\end{array}\right)} \text {, and }
\end{aligned}
$$

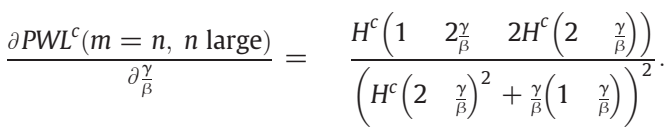

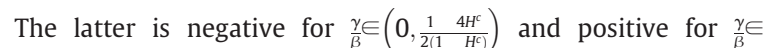

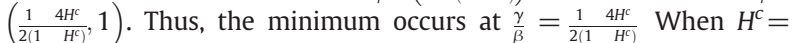
0.18 , which the FTC considers the threshold for a concentrated industry, the minimal $P W L^{c}$ is 0.241967 . This value is a large lower bound.

Now we consider welfare losses in a Bertrand equilibrium.

Lemma 4. In a Bertrand equilibrium with heterogeneous firms

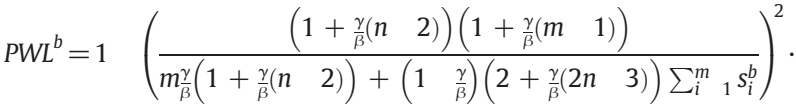

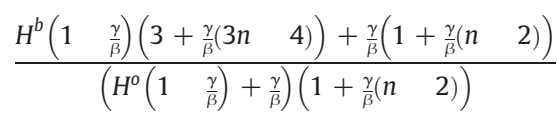

Proof. Social welfare in a Bertrand equilibrium, denoted by $W^{b}$, is given by

$W^{b}=\frac{(\beta \quad \gamma)(3 \beta+\gamma(3 n}{(3))} H^{b} x^{b 2}+\frac{\gamma}{2} x^{b 2}$.

Let $P W L^{b}$ be the percentage of welfare losses in a Bertrand equilibrium.

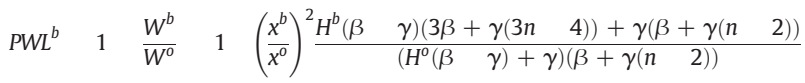

Plugging in the values of $x^{b}$ and $x^{o}$, we obtain the formula above. $\square$

Thus, $P W L^{b}$ depends on the degree of product differentiation $\frac{\gamma}{\beta}$, the number of active firms in the optimum $m$ and in a Bertrand equilibrium $n$, the sum of the market shares of the $m$ largest firms $\sum_{i}{ }^{m}{ }_{1} s_{i}^{b}$, and the Hirschman Herfindahl indices of concentration $H^{b}$ 
and $H^{\circ}$, evaluated at a Bertrand equilibrium and in the optimum respectively.

As before, let us consider two special cases. First, when in the optimum only firm 1 is used by the planner. Thus, $m=1$ and

$$
\begin{aligned}
& P W L^{b}(m=1)=1 \quad\left(\frac{1+\frac{\gamma}{\beta}\left(\begin{array}{ll}
n & 2
\end{array}\right)}{\frac{\gamma}{\beta}\left(1+\frac{\gamma}{\beta}\left(\begin{array}{ll}
n & 2
\end{array}\right)\right)+\left(\begin{array}{ll}
1 & \frac{\gamma}{\beta}
\end{array}\right)\left(2+\frac{\gamma}{\beta}\left(\begin{array}{ll}
2 n & 3
\end{array}\right)\right) s_{1}^{b}}\right)^{2}
\end{aligned}
$$

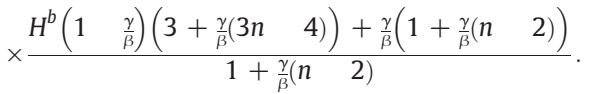

For $\beta \simeq \gamma$, this formula reduces to $P W L^{b}(m=1)=0$, as one expects for a Bertrand equilibrium under product homogeneity. Notice that $\operatorname{PWL}^{b}(m=1)$ is decreasing in $H^{b}$. Second, when the number of active firms is the same in the optimum and in a Bertrand equilibrium, after lengthy calculations, we obtain the result

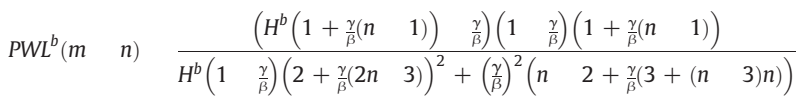

If all firms are identical, $H^{b} \quad \frac{1}{n}$ and $\operatorname{PWL}^{b}(m \quad n) \quad\left(\frac{1-\frac{\gamma}{\gamma}}{2+\frac{\gamma}{\beta}(n-3)}\right)^{2}$ as in Lemma 2. Finally, when $n$ is large, (2.18) simplifies to

$P W L^{b}(m=n, n$ large $)=\frac{H^{b}\left(\begin{array}{ll}1 & \frac{\gamma}{\beta}\end{array}\right)}{\frac{\gamma}{\beta}+4 H^{b}\left(\begin{array}{ll}1 & \frac{\gamma}{\beta}\end{array}\right)}$

which is decreasing in the degree of product differentiation $\frac{\gamma}{\beta}$. Its maximal value is 0.25 (for $\frac{\gamma}{\beta}=0$ ). For $H^{b}=0.18, P W L^{b}(m=$ $n, n$ large $)=\frac{0.18 \quad 0.18^{\gamma}}{0.28_{\beta}^{\gamma}+0.72}$ which for values of $\frac{\gamma}{\beta}$ larger than 0.75 is less than $4.8 \%$. So in this case a high concentration does not imply large welfare losses.

From (2.16) and (2.18) we obtain the following result:

Proposition 7. $\operatorname{PWL}^{j}(m=n)$ is increasing in $H^{j}, j=c, b$.

Proof. Computing $\frac{\partial P W L^{c}}{\partial H^{c}}(m=n)$, we obtain

$\frac{\frac{\gamma}{\beta}\left(\begin{array}{ll}1 & \frac{\gamma}{\beta}\end{array}\right)\left(\begin{array}{lll}1+\frac{\gamma}{\beta}(n & 1\end{array}\right)\left(\begin{array}{ll}4+\frac{\gamma}{\beta}(n & 2\end{array}\right)}{\left.\left(\begin{array}{lll}4 H^{c}\left(1+\frac{\gamma}{\beta}(n\right. & 2\end{array}\right)\right)+\left(\frac{\gamma}{\beta}\right)^{3}\left(\begin{array}{lll}H^{c} & 1\end{array}\right)\left(\begin{array}{ll}n & 1\end{array}\right)+\left(\frac{\gamma}{\beta}\right)^{2}\left(\begin{array}{lll}n & \left.2+H^{c}\left(\begin{array}{ll}5 & 4 n\end{array}\right)\right)^{2}\end{array}\right.}$,

which is positive for $\frac{\gamma}{\beta}>0$. Also, $\frac{\partial P L^{b}}{\partial H^{b}}(m=n)$ is equal to

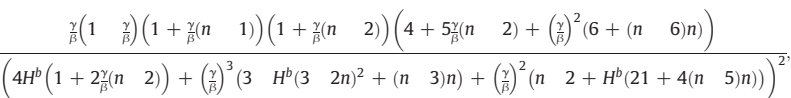

which is positive for $\frac{\gamma}{\beta}>0$.

Thus, for $m=n P W L$ increases with $H$, contrary to what happens when $m=1$ in both Cournot and Bertrand equilibria. This is because the condition $m=n(m=1)$ is related to goods being poor (good) substitutes. Finally, a word of caution: in Proposition 7 we have assumed that $H$ is independent of all other variables affecting PWL, for example $\frac{\gamma}{\beta}$ and $n$. But $H$ does depend on these variables. Strictly speaking, Proposition 7 only applies to variations in $H$ that are caused by variations in the $\alpha$ 's and $c^{\prime} s$.

\section{A model of a large group}

In this section we consider the market for a differentiated good supplied by a large number of firms. Typical examples include restaurants, wine, beer, etc. We will not consider entry and fixed costs, which as shown in Corchón (2008) might produce a very high
PWL and bias our estimates. As the purpose of this paper is to study the impact of product differentiation alone, we discard these costs. As we will see, even so this model is capable of producing a very high PWL. The model can be interpreted as a monopolistic competition model in which the long run aspects are not considered. In this framework the relative size of firms is not an important issue, so we will assume that all firms are identical. Also, for convenience, we will assume that firms compete in quantities.

The consumer surplus is given by

$U=\left(\sum_{i=1}^{n} x_{i}^{\delta}\right)^{\frac{r}{\delta}} \quad \sum_{i}^{n} p_{i} x_{i}, \delta, r \in(0,1)$

(see Spence, 1976). The inverse demand function of firm $i$ is $p_{i}=$ $r\left(\sum_{i}^{n}{ }_{1} x_{i}^{\delta}\right)^{\bar{\delta}} 1 x_{i}^{\delta} \quad 1$.

Definition 5. A CES market is a list $\{\delta, r, c, n\}$ with $\delta, r \in(0,1), c<0$, and $n \in \mathbb{N}$.

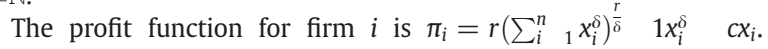
Because there are many firms, each firm takes $\sum_{i}{ }^{n}{ }_{1} x_{i}^{\delta}$ as given. The elasticity of demand, denoted by $\varepsilon$, is defined as the inverse of the elasticity of inverse demand:

$\epsilon=\frac{1}{1 \delta}$

Thus, as $\delta \rightarrow 1$ the elasticity of demand becomes infinite. Now we have the following preliminary result.

Lemma 5. In a CES market

$P W L^{S}=1 \quad \delta^{\frac{1}{1-\frac{1}{\delta}}} \frac{r}{1} \quad r$

Proof. The FOC of profit maximization for firm $i$ is

$r\left(\sum_{i}^{n} x_{i}^{\delta}\right)^{\frac{r}{\delta}} \quad 1 \quad \delta x_{i}^{\delta} \quad 1 \quad c=0$.

The left hand side of (3.3) is decreasing in $x_{i}$, so the second order condition holds. In a symmetric equilibrium where all firms produce the same output, denoted by $x_{i}^{*}$, we have

$x_{i}^{*}=\left(\frac{r \delta}{c n^{1} \frac{r}{\delta}}\right)^{\frac{1}{1 r}}, p^{*}=\frac{c}{\delta}$ and $U^{*}=n^{\frac{r}{\delta}}\left(\frac{r \delta}{c n^{1} \frac{r}{\delta}}\right)^{\frac{r}{1 r}}$.

In this equilibrium, the social welfare is

$W^{*}=n^{\frac{r}{\delta}}\left(\frac{r \delta}{c n^{1} \frac{r}{\delta}}\right)^{\frac{r}{1 r}} n c\left(\frac{r \delta}{c n^{1} \frac{r}{\delta}}\right)^{\frac{1}{1 r}}$

In the optimum the price equals the marginal cost. Thus, $r\left(\sum_{i}^{n}{ }_{1} x_{i}^{\delta}\right)^{\frac{r}{\delta}}{ }^{\frac{1}{2}} x_{i}^{\delta} \quad{ }^{1}=c$. From this we get

$x_{i}^{o}=\left(\frac{r}{c n^{1} \frac{r}{\delta}}\right)^{\frac{1}{1 r}}$ and $W^{0}=n^{\frac{r}{\delta}}\left(\frac{r}{c n^{1} \frac{r}{\delta}}\right)^{\frac{r}{1-r}} n c\left(\frac{r}{c n^{1} \frac{r}{\delta}}\right)^{\frac{1}{1 r}}$,

where $x_{i}^{o}$ and $W^{o}$ stand for the output and social welfare in the optimum. $W^{o}$ is increasing in $n$, so in the full optimum the planner would choose a number of firms equal to $n$. 
Consequently, the percentage of welfare losses is:

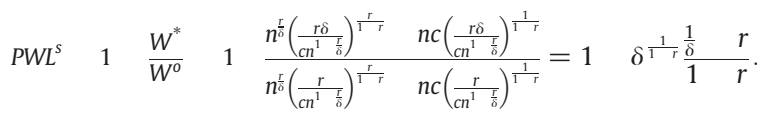

At first glance it is surprising that $P W L^{S}$ does not depend on the number of firms $n$. However, we did assume that the number of firms is large. Thus, (3.2) can be understood as the limit formula when $n$ is large. The following properties of $P W L^{s}$ are easily proven:

Proposition 8. i) $P W L^{s}$ is decreasing in $\delta$.

ii) $\lim _{\delta \rightarrow 1} P W L^{S}=0$ and $\lim _{\delta \rightarrow 0} P W L^{S}=1$.

iii) $P W L^{s}$ is increasing in $r$.

iv) $\lim _{r \rightarrow 1} P W L^{S}=1$ and $\lim _{r \rightarrow 0} P W L^{S}=0$.

The explanation of ii) is that when $\delta$ is close to one (resp. zero), the products are close to being homogeneous (resp. very differentiated), and welfare losses are small (resp. large); see formula (3.1). The explanation of iii) is that as $r$ increases (resp. decreases), the gap between the optimal and the equilibrium output increases (resp. decreases); see formulae (3.4) and (3.5). It follows from ii) and iv) that it is possible to have a market where the elasticity of demand is close to infinity (i.e., $\delta$ is close to 1 ) and PWL is as close to 1 as we wish. ${ }^{10}$ In brief, elasticity of demand is only a partial measure of PWL in this model.

Let us relate $P W L^{s}$ to the observable variables listed in Definition 4 of the previous section. The FOC of profit maximization imply that $\epsilon=\frac{p}{p}$, so in this framework, as in the Bertrand case in the previous section, knowledge of the elasticity of demand is of no help. We will assume that $\mathfrak{c}(\ln \mathfrak{n}+\ln \mathfrak{p})<\mathfrak{p} \ln \mathfrak{n}$; this condition ensures that $r<1$.

In our construction, the function ProductLog $(t)$ will play a prominent role. This function, called Lambert's $W$ function, gives the solution for $w$ in $t=w e^{w}$ and has the following properties: ${ }^{11}$

i) ProductLog $(t) \in \mathbb{R}$ for $t \in\left[\frac{1}{e}, \infty\right)$;

ii) $\operatorname{ProductLog}\left(\frac{1}{e}\right)=1$;

iii) $\lim _{\delta \rightarrow \infty}$ ProductLog $(t)=\infty$;

iv) ProductLog $(0)=0$;

v) ProductLog $(t)$ is increasing in $t \in\left[\frac{1}{e}, \infty\right)$;

vi) $e^{a \text { ProductLog(t) }}(\text { ProductLog }(t))^{a}=t^{a}$.

Now we arrive at the main result of this section:

Proposition 9. Given an observation $\left\{\mathfrak{p}, \mathfrak{x}_{i}, \mathfrak{c}, \mathfrak{n}\right\}$ there is a CES market $\{\delta, r, \mathfrak{c}, \mathfrak{n}\}$ such that $\left(\mathfrak{p}, \mathfrak{x}_{i}\right)$ is an equilibrium for this market, and

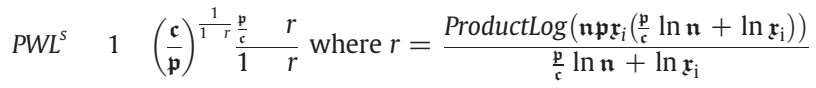

Proof. Let $\delta$ and $r$ be such that

$\left(\frac{r \delta}{\mathfrak{c n}^{1} \frac{r}{\delta}}\right)^{\frac{1}{1} r}=\mathfrak{x}_{i}$ and $\frac{\mathfrak{c}}{\delta}=\mathfrak{p}$

The previous equations yield

$\delta=\frac{\mathfrak{c}}{\mathfrak{p}}$ and $r=\frac{\text { ProductLog }\left(\mathfrak{n} \mathfrak{p} \mathfrak{x}_{i}\left(\frac{\mathfrak{p}}{\mathfrak{c}} \ln \mathfrak{n}+\ln \mathfrak{x}_{\mathfrak{i}}\right)\right)}{\frac{\mathfrak{p}}{\mathfrak{c}} \ln \mathfrak{n}+\ln \mathfrak{x}_{\mathrm{i}}}$

It is straightforward to check that $0<\delta<1$ and $0<r<1$ (using the condition $\frac{\mathfrak{c}}{\mathfrak{p}}<\frac{\ln \mathfrak{n}}{\ln \mathfrak{n}+\ln \mathfrak{p})}$. Then by construction the CES market $\{\delta, r, \mathfrak{c}, \mathfrak{n}\}$ yields an equilibrium where $p^{*}=\mathfrak{p}$ and $x_{i}^{*}=\mathfrak{x}_{i}$. Plugging $\delta$ and $r$ into (3.2), we get the formula for $P W L^{s}$ as a function of an observation $\left\{\mathfrak{p}, \mathfrak{x}_{i}, \mathfrak{c}, \mathfrak{n}\right\}$.

\footnotetext{
${ }^{10}$ Even if $\delta=r$, $\lim _{\delta \rightarrow 1} P W L^{s}=0.2642$, a large number

11 See Weisstein (1999).
}

An important consequence of Proposition 9 is that, given an observation, there is a unique value of $P W L^{S}$. In this case, the number of parameters to be "recovered" is equal to the number of data.

Next, we analyze the properties of $P W L^{S}$ in (3.6):

Proposition 10. The percentage of welfare losses in the CES model is such that

i) $\lim _{\mathfrak{n} \rightarrow \infty} P W L^{s}=1 \quad\left(\frac{\mathfrak{c}}{\mathfrak{p}}\right)^{\frac{1}{1}}\left(\frac{\mathfrak{p}}{\mathfrak{c}}+1\right)$.

ii) $\lim _{\underline{\mathbf{c}} \rightarrow 1} P W L^{S}=0$.

iii) $\lim _{\overline{\mathfrak{p}} \rightarrow 1}^{\stackrel{\mathfrak{p}}{\mathfrak{c}_{1} \rightarrow 1}}\left(\lim _{\mathfrak{n} \rightarrow \infty} P W L^{S}\right)=1 \quad \frac{2}{e} \simeq 0.2642$.

Note that when a finite number of firms are pricing at the marginal cost, $P W L^{s}$ is close to zero. When an infinite number of firms are pricing at the marginal cost, however, $P W L^{s}$ is quite high. In fact, it could be argued that formula i) above should be used since we assumed that $n$ was large. In this case, $P W L^{s}$ is decreasing in the price marginal cost margin, $\frac{\mathfrak{p}, \mathfrak{p}}{\mathfrak{p}} .12$

\section{Conclusion}

In this paper we have studied the relationship between observable variables and welfare losses, taking the behavior of firms as given. ${ }^{13}$ Our main message is positive in that relating welfare losses to observables is a feasible endeavor in the models considered in this paper. ${ }^{14}$ But there is an important caveat: the calculus of welfare losses depends on the forms of demand and costs not only at the equilibrium point, but at all points in the domain of these functions. This contrasts with the typical linearization around the equilibrium where a linear form is supposed to represent the characteristics of a general function around the equilibrium point. It is clear that welfare losses depend in a fundamental way on the functional forms, so this issue cannot be dodged. Thus, one can interpret our results by saying that they show welfare consequences of assumptions that are often made about the functional forms of demand and costs.

We end this paper by giving some hints as to how data and elasticities may help us to discriminate among the models. The clearest case is a Bertrand equilibrium. A necessary condition for this equilibrium to be supported by the data is that for all $i, p_{i}=\varepsilon\left(p_{i} \quad c_{i}\right)$ (irrespective of whether the market is linear). If the elasticity of demand cannot be estimated, Proposition 2 says that any observation can be interpreted as a Bertrand equilibrium. The case for the CES model relies on two assumptions. On the one hand, the elasticity of demand must be constant. On the other hand, the cross elasticity of demand (calculated

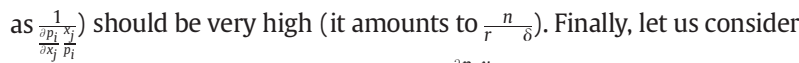
the Cournot equilibrium. Let $\xi \equiv \frac{\partial p_{i}}{\partial x_{i}} \frac{x_{i}}{p_{i}}$ be the elasticity of the inverse demand function. From the FOC of profit maximization, we have $\xi p_{i}=p_{i} \quad c_{i}$. The elasticity $\xi$ can be obtained by inverting the system of demand functions. For instance, in the symmetric case with $n=2, \xi=\frac{\varepsilon}{\varepsilon^{2}} \rho^{2}$, which when plugged into the FOC yields $\frac{p_{i} c_{i}}{p_{i}}=$ $\frac{\varepsilon}{\varepsilon^{2}} \rho^{2}$. If markups, demand and cross elasticities can be estimated, they must obey the previous equation in a Cournot equilibrium.

\section{References}

Amir, R., Jin, J.Y., 2001. Cournot and Bertrand equilibria compared: substitutability, complementarity and concavity. International Journal of Industrial Organization 19 303-317.

${ }^{12}$ When $n$ is not large, we have an example, available upon request, showing that PWL is not monotonic in the price-marginal cost margin.

${ }^{13}$ See Sutton (1998) for an approach where the only source of variation between firms is the degree of competitiveness.

${ }^{14}$ The models presented in this paper have been selected for their impact in the profession. Thus, the papers by Dixit, Singh and Vives and Spence obtained in the aggregate nearly 1800 citations in Google Scholar. 
Anderson, S.P., Renault, R., 2003. Efficiency and surplus bounds in Cournot competition. Journal of Economic Theory 113, 253-264.

Cable, J., Carruth, A., Dixit, A., 1994. In: Cable, J. (Ed.), Oligopoly and Welfare in Current ssues in Industrial Economics. InMacmillan Press, London.

Coate, M., 2005. Empirical analysis of merger enforcement under the 1992 Merger Guidelines. Review of Industrial Organization 27 (4), 279-301.

Corchón, L.C., 2008. Welfare losses under Cournot competition. International Journal of Industrial Organization 26, 1120-1131.

Corchón, L.C., Zudenkova, G., 2008. Computing Welfare Losses from Data under Imperfect Competition with Heterogeneous Goods. UC3M WP 08-26 (16).

Cowling, K., Mueller, D.C., 1978. The social costs of monopoly power. Economic Journal $88,727-748$

Daughety, A.,1990. Beneficial concentration. American Economic Review 80,1231-1237. Dixit, A.K. 1979. A model of duopoly suggesting a theory of entry barriers. Bell Journal of Economics 10, 20-32.

Dixit, A.K., Stiglitz, J.E., 1977. Monopolistic competition and optimum product diversity. American Economic Review 67, 297-308.

Farrell, J., Shapiro, C., 1990. Horizontal merger: an equilibrium analysis. American Economic Review 80, 107-126.

Formby, J., Layson, S., 1982. Allocative inefficiency and measures of market power. Atlantic Economic Journal 10 (4), 67-70.

Häckner, J., 2000. A note on price and quantity competition in differentiated oligopolies. Journal of Economic Theory 93 (2), 233-239.
Harberger, A.C., 1954. Monopoly and resource allocation. American Economic Review: Papers and Proceedings 44, 77-87.

Hsu, J., Wang, X.H., 2005. On welfare under Cournot and Bertrand competition in differentiated oligopolies. Review of Industrial Organization 27 (2), 185-191.

Johari, R. Tsitsiklis, J. 2005. Efficiency loss in Cournot games, Mimeo, Jan. 28.

Lahiri, S., Ono, Y., 1988. Helping minor firms reduces welfare. Economic Journal 98, $1199-1202$.

Lerner, A., 1934. The concept of monopoly and the measurement of monopoly power. Review of Economic Studies 1 (3), 157-175.

McHardy, J.P., 2000. Miscalculations of monopoly and oligopoly welfare losses with linear demand. Hull Economic Research Papers. November.

Singh, N., Vives, X., 1984. Price and quantity competition in a differentiated oligopoly. Rand Journal of Economics 15, 546-554.

Spence, M., 1976. Product selection, fixed costs, and monopolistic competition. Review of Economic Studies 43, 217-235.

Stigler, G.J., 1949. Monopolistic Competition in Retrospect in Five Lectures on Economic Problems. Longmans, Green and Co, London.

Sutton, J., 1998. Technology and Market Structure. MIT Press, Cambridge, MA.

Weisstein, E.W., 1999. The CRC Concise Encyclopedia of Mathematics. CRC Press LLC. 